\title{
Contrasting SARS-CoV-2 epidemics in Singapore: Cohort studies in migrant workers and the general population
}

\section{Hannah E Clapham}

National University of Singapore

Wan Ni Chia

Duke-NUS Graduate Medical School

Linda Wei Lin Tan

National University of Singapore

Vishakha Kumar

National University of Singapore

Jane M Lim

National University of Singapore

Nivedita Shankar

National University of Singapore

Zaw Myo Tun

National University of Singapore

Marina Zahari

National University of Singapore

Li Yang Hsu

National University of Singapore

Louisa J Sun

National University Health System

Linfa Wang

Duke-NUS Graduate Medical School

Clarence C Tam ( $\nabla$ clarence.tam@nus.edu.sg)

National University of Singapore

\section{Research Article}

Keywords: SARS-CoV-2, COVID-19, serological studies, longitudinal studies, disease incidence, disease burden, respiratory infections

Posted Date: April 20th, 2021 
DOl: https://doi.org/10.21203/rs.3.rs-441527/v1

License: (c) (1) This work is licensed under a Creative Commons Attribution 4.0 International License. Read Full License

Version of Record: A version of this preprint was published at International Journal of Infectious Diseases on December 1st, 2021. See the published version at https://doi.org/10.1016/j.ijid.2021.11.043. 


\section{Abstract}

From January 2020, Singapore implemented comprehensive measures to suppress SARS-CoV-2. Community transmission has been limited, although explosive outbreaks have occurred in migrant worker dormitories. We conducted longitudinal SARS-CoV-2 serology studies among 478 residents of a SARSCoV-2 affected migrant worker dormitory between May and July 2020, and 937 community-dwelling adult Singapore residents with sera collected before September 2019 and in November/December 2020. By end 2020, <2 per $1000(0.16 \%, 95 \%$ Crl: $0.008 \%-0.72 \%)$ adult residents in the community were infected with SARS-CoV-2, approximately 4 times higher than the national notified case incidence. In contrast, in the migrant worker cohort, nearly two-thirds $63.8 \%$ (95\% Crl: $57.9 \%-70.3 \%$ ) had been infected by July 2020 ; no symptoms were reported in $>90 \%$ of these infections. SARS-CoV-2 suppression is feasible with rapid implementation of comprehensive control measures. However, the risk of large-scale epidemics in densely-populated environments requires specific consideration in preparedness planning.

\section{Introduction}

Singapore reported its first imported case of Coronavirus Disease 2019 (COVID-19) on 23 January 2020. The first cases of local transmission were recorded on 4 February 2020. Since then, Singapore has experienced three distinct COVID-19 epidemics, involving imported cases, clusters of community transmission linked mainly to workplaces, social gatherings and nursing homes, and outbreaks among migrant workers (Figure 1). The public health response has involved a comprehensive set of control measures, including border restrictions, isolation of COVID-19 cases in hospitals or dedicated government facilities, extensive manual and digital contact tracing, and strict quarantining and testing of incoming travellers and exposed individuals. In addition, extensive restrictions on social gatherings and universal mask wearing have been in place since April 2020, when an eight-week national lockdown was initiated.

These measures have largely been effective at curtailing community transmission of SARS-CoV-2. However, from March 2020, Singapore experienced a series of large epidemics in densely populated migrant worker dormitories. ${ }^{1}$ In response, workers were confined to dormitories during the lockdown period and movement within dormitories was restricted. Medical teams were deployed in all dormitories to provide testing and refer suspected COVID-19 cases to healthcare facilities, leading to the eventual interruption of transmission in September 2020. As of 16 March 2021, 60,117 confirmed COVID-19 cases have been reported nationally. Of these, $>90 \%$ have been among male foreign migrant workers living in dormitories. Here, we report on the contrasting SARS-CoV-2 epidemics among migrant workers and the community in Singapore, based on two longitudinal serological studies.

\section{Methods}

Migrant worker cohort 
Between May and July 2020, we conducted a longitudinal serology study among 541 male residents of a migrant worker dormitory in Singapore. At the start of the epidemic, the dormitory housed over 4000 residents of primarily Indian, Bangladeshi and Chinese origin working mainly in the construction, shipping, manufacturing and processing sectors. The dormitory consisted of 10 multi-storey residential blocks, with rooms typically housing 10-14 residents. At the time of the study, clinical management and testing of suspected COVID-19 cases within the dormitory was provided by a mobile medical team. PCRconfirmed COVID-19 cases were referred to acute care hospitals or community isolation facilities, depending on medical need.

We recruited participants from randomly selected rooms within each residential block ( 5 rooms per block). Consenting participants provided a $5 \mathrm{ml}$ venous blood sample at enrollment and after two and six weeks. We collected information on participants' demographics, country of origin, work sector, preexisting health conditions and smoking history at enrollment. Additionally, we asked participants whether they had experienced any symptoms compatible with COVID-19 in the month prior to enrollment and in the intervening periods between follow-up visits. COVID-19 compatible symptoms included fever, cough, shortness of breath, sore throat, runny nose, anosmia, muscle ache, fatigue and diarrhoea.

\section{Community cohort}

Participants were recruited from among individuals taking part in the Singapore Population Health Studies (SPHS), which comprises 50,000 adult Singaporeans and long-term residents followed up over multiple waves to monitor risk factors for common health conditions. Individuals in SPHS were eligible to take part in this study if they were Singapore Citizens or Permanent Residents aged $>=21$ years and had a stored serum sample collected prior to between January and August 2019 available for serological testing. Participants were recruited between November and December 2020. Trained interviewers conducted an interview by telephone to collect demographic details and information on potential risk factors for SARS-CoV-2 infection, including occupation, travel history, contact with suspected or confirmed COVID-19 cases, and co-morbidities. An in-person visit was subsequently arranged within 7 days, at which participants provided a $5 \mathrm{ml}$ venous blood sample for serological testing.

\section{Serological testing}

We tested for the presence of SARS-CoV-2 neutralising antibodies in pre-pandemic sera and sera collected in November/December 2020 from all community cohort participants, and in sera collected at baseline, two and six weeks from the migrant worker cohort. Sera were extracted from fresh blood samples within 24 hours of collection. We tested for SARS-CoV-2 neutralising antibodies (NAb) using the cPass SARS-CoV-2 Neutralization Antibody Detection Kit (GenScript). An inhibition threshold of $30 \%$ was used to define a positive result, as per the manufacturer's instructions.

\section{Data analysis}


We estimated cumulative seroprevalence in November/December 2020 in the community cohort using a Bayesian approach accounting for test sensitivity and specificity, as described by Larremore et al. $^{2} \mathrm{We}$ obtained 5000 samples from the posterior parameter distributions Markov Chain Monte Carlo (MCMC) methods, after a burn-in of 1000 iterations.

For the migrant worker cohort, we estimated cumulative seroprevalence at baseline, two and six weeks of follow-up using a Bayesian random effects logistic model as described by Stringhini et al. ${ }^{3}$ (Supplementary Information), which additionally accounts for the clustering of observations from residents sampled from the same room. In addition, we estimated the risk of seroconversion during the follow-up period among individuals who were seronegative at baseline. We obtained 6000 samples from the posterior parameter distributions using four MCMC chains, each with a burn-in of 750 iterations. We checked for model convergence using the R-hat statistic and through visual inspection of trace plots to ensure good mixing of MCMC chains.

Information on false positivity and false negativity for the cPass assay were obtained from a validation study by Meyer et al., ${ }^{4}$ which estimated a sensitivity $80.3 \%$ and specificity of $99.3 \%$ respectively.

Analyses were conducted in R software version 4.1. ${ }^{5}$ Bayesian random effects models were fitted in Stan using the rstan package. ${ }^{6}$ We summarised the posterior parameter distributions using the median and central $95 \%$ of the posterior distribution.

\section{Ethics statement}

The community cohort study was approved by the National University of Singapore Institutional Review Board (reference H-20-032). Participants in this study provided signed, informed consent. The migrant worker cohort study was approved by the Singapore Ministry of Health under the Infectious Diseases Act and was exempt from institutional review board approval as it was conducted as part of the national public health response to the COVID-19 pandemic. Participants provided verbal consent to take part.

\section{Results}

\section{Participation}

Of 551 individuals approached, we recruited 541 (98.2\%) participants in the male migrant worker cohort. Of these, 478 (88.4\%) provided 3 blood samples at recruitment, two and six weeks, and were included in this analysis. The mean age was 35 years (range: $19-59$ years). Among the participants, $51.4 \%$ originated from India, 34.9\% from Bangladesh and 9.4\% from China. Nearly a third of participants were current smokers, but underlying medical conditions were uncommon (Table 1).

In the community cohort, we approached 2608 individuals of whom 701 (26.9\%) were not contactable after three attempts and three $(0.1 \%)$ were no longer in Singapore or had passed away. Of the remaining 1904 individuals, 937 (49.2\%) agreed to participate and completed the baseline assessment. Participants 
had a mean age of 52 years (range: $23-83$ years) and 480 (51.2\%) were female. The most common underlying conditions were high blood pressure (23.2\%), diabetes $(15.0 \%)$ and chronic respiratory conditions (6.5\%); $9.7 \%$ were current smokers (Table 1 ).

Recruitment summaries for the two cohorts are shown in the Supplementary Information.

\section{Seroprevalence and infection risk}

In the migrant worker cohort, 117 (24.5\%) were positive for SARS-CoV-2 neutralising antibodies at baseline. This rose to 178 (37.2\%) after two weeks and 245 (51.3\%) after six weeks. After accounting for test characteristics, the cumulative seroprevalence was estimated to be $30.4 \%$ (95\% Crl: $26.1 \%$ - 35.9\%) at baseline, $46.5 \%$ (95\% Crl: $41.4 \%$ - 51.9\%) after two weeks and 63.8\% (95\% Crl: 57.9\% - 70.3\%) after six weeks. Among initially seronegative migrant workers, the risk of seroconversion was $22.9 \%$ (95\% Crl: $18.5 \%-27.8 \%$ ) by two weeks and $54.3 \%$ (95\% Crl: $48.3 \%-60.7 \%$ ) by week six of follow-up (Figure 2 ).

There was statistical and epidemiological evidence that infections clustered strongly by room (household random effect at baseline, $\sigma_{\mathrm{h}}=3.0,95 \% \mathrm{Crl}$ : $\left.1.93-5.27\right)$. This clustering effect decreased over time as seroprevalence increased over the six-week follow-up period ( $\sigma_{h}=1.74,95 \%$ Crl: $1.11-2.86$ at six weeks). Additionally, among 16 initially seronegative rooms widely heterogeneous trajectories were observed (Multimedia File), with attack rates over the six-week follow-up ranging from $0 \%$ to $85 \%$ (Supplementary Information).

The vast majority of migrant worker infections were asymptomatic. Among those seropositive at baseline, five (4.3\%) reported experiencing any symptom compatible with COVID-19 in the previous four weeks, and $3.8 \%(6 / 160)$ of seroconverters reported symptoms during the six week follow-up period. We found no association between seropositivity at baseline or seroconversion and age, country of origin, work sector, occurrence of symptoms or smoking status.

In the community cohort, no pre-pandemic serum samples were positive for SARS-CoV-2 neutralising antibodies. Among samples collected in November/December 2020, 0.21\% (2/937) tested positive. Both positive samples were non-reactive against SARS virus spike protein. After accounting for test characteristics, we estimated the cumulative seroprevalence in 2020 to be $0.16 \%$ (95\% Crl: $0.008 \%$ $0.72 \%)$.

A total of 2272 community cases of COVID-19 were reported in Singapore up to the end of December 2020, corresponding to a cumulative incidence of $0.042 \% .{ }^{7}$ This indicates a most likely value for the infection:case ratio of 3.8:1.

\section{Discussion}

Our findings highlight the contrasting epidemics in the community and in migrant worker populations in Singapore, based on serological evidence from two longitudinal studies. These studies demonstrate the 
continuing effectiveness of nationally-implemented, wide-ranging control measures for minimising community transmission of SARS-CoV-2, while emphasising the challenges to mitigating risk in densely populated settings. We also documented evidence of high rates of asymptomatic infection in migrant workers.

To our knowledge, this is the first study to report on SARS-CoV-2 cross-reactivity in pre-pandemic sera from a large, systematic, general population cohort. We found no evidence of population exposure to SARS-CoV-2 prior to September 2019. Since January 2020, Singapore has implemented a multi-pronged strategy for SARS-CoV-2 containment and suppression, including extensive testing and contact tracing, strictly enforced case isolation and quarantining, and risk-based border restrictions, social distancing measures and mask-wearing policies. Early modelling work in Singapore emphasised the important role of case isolation, contact tracing, quarantining and workplace distancing measures in controlling SARSCoV-2 transmission, ${ }^{8}$ which is evidenced by the comparatively small number of community COVID-19 cases notified to date and the extremely low seroprevalence observed in this study. In comparison, early seroprevalence studies in European settings indicated that between $5 \%-10 \%$ of the population had evidence of SARS-CoV-2 exposure after the first epidemic wave. ${ }^{3,9}$

Initial measures in Singapore, however, were unable to prevent large epidemics in densely populated migrant worker dormitories. Data from our study indicated that around two-thirds of dormitory residents had been infected between April and early July, in line with seroprevalence estimates reported from other migrant worker populations. ${ }^{1}$ Moreover, our data suggest extremely high infection risks in these settings, with more than half of initially immunologically naive individuals becoming infected over the six-week follow-up period. Crowded, poorly ventilated indoor spaces are known to be high-risk environments for SARS-CoV-2 transmission, and have been associated with large outbreaks among processing plant workers. ${ }^{10-12}$

Despite this, the vast majority of infections were asymptomatic. This finding should be interpreted in the context of prevailing control measures in migrant worker dormitories. Individuals at higher risk of severe COVID-19, including older individuals and those with underlying medical conditions, were moved to alternative housing early on in the epidemic. Additionally, universal mask-wearing was mandatory during the study period, which is likely to have contributed to reducing droplet exposure from infectious individuals and, hence, viral inoculum. ${ }^{13}$ Although it is possible that participants may have under-reported symptoms, we believe this is an unlikely explanation for the high fraction of asymptomatic infections. At the time of the study, dormitory residents were required to report their temperature every day and were asked to report to a medical post within the dormitory in the event of symptoms for testing and referral to medical services where necessary. All but essential work had been suspended and residents were confined to the dormitory. The Singapore government set up a compensation scheme so that workers continued to be paid during this period. It is thus unlikely that there were strong disincentives to report symptoms. 
Based on a comparison of cumulative seroprevalence in our community cohort to officially reported COVID-19 cases in the community up to the end of 2020, we estimate a likely value for the infection:case ratio of around $4: 1$, although uncertainty is high because of the very small number of seroconversions. Importantly, Singapore has employed extensive testing, case identification and contact tracing since the start of the pandemic, and ascertainment of community COVID-19 cases is likely to be near complete. Our estimate for the infection:case ratio is in agreement with analyses of the Diamond Princess cruise ship outbreak, in which an estimated three-quarters of infections were asymptomatic. ${ }^{14}$ Data from extensive investigations of the wider outbreak in migrant workers also identified 5 times as many infections as symptomatic cases, ${ }^{1}$ although there may have been some under-ascertainment of symptomatic cases among migrant workers early in the epidemic.

Within the broader context of the COVID-19 epidemic in Singapore, our findings demonstrate that suppression of SARS-CoV-2 transmission is possible with strict and rapid implementation of border restrictions, case isolation, contact tracing, quarantining and social distancing measures. However, the potential for large-scale epidemics in densely-populated environments means that these high-risk settings require special consideration in preparedness, mitigation and control planning. Consideration and prioritisation of these groups in vaccination strategies should help minimise risk of future resurgences and potential spillover of transmission to the wider community.

\section{Declarations}

\section{Author contributions}

Drs Tam and Clapham had full access to all of the data in the study and take responsibility for the integrity of the data and the accuracy of the data analysis.

Concept and design: Tam, Clapham.

Acquisition, analysis, or interpretation of data: All authors.

Drafting of the manuscript: Tam, Clapham.

Critical revision of the manuscript for important intellectual content: All authors.

Statistical analysis: Tam, Clapham.

Administrative, technical, or material support: Tan, Lim, Shankar, Zahari, Kumar, Tun.

Acknowledgements

Recruitment and data collection for the two cohort studies was made possible by the Singapore Population Health Studies operations team.

Sources of funding 
The community cohort study is supported by a Wellcome Trust grant (grant number: 221013/Z/20/Z). The migrant worker cohort study is supported by the NUS Efforts Against COVID-19 fund. The serological test development at Duke-NUS was supported by grants from the Singapore National Medical Research Council (STPRG-FY19-001 and COVID19RF-003) .

Competing interests statement:

L-FW and WNC are co-inventors of a patent application for the cPass test kit. The remaining authors declare no competing interests in relation to this work.

Author biography:

Dr. Clapham is an assistant professor in the Saw Swee Hock School of Public Health at the National University of Singapore. Her research covers mathematical modelling of infectious diseases, including the design and use of seroepidemiologic studies and surveillance to understand disease transmission.

\section{References}

1. Tan IB, Tan C, Hsu LY, et al. Prevalence and Outcomes of SARS-CoV-2 Infection Among Migrant Workers in Singapore. JAMA. 2021;325(6):584-585. doi:10.1001/jama.2020.24071

2. Larremore DB, Fosdick BK, Bubar KM, et al. Estimating SARS-CoV-2 seroprevalence and epidemiological parameters with uncertainty from serological surveys. Elife. 2021;10. doi:10.7554/eLife.64206

3. Stringhini S, Wisniak A, Piumatti G, et al. Seroprevalence of anti-SARS-CoV-2 IgG antibodies in Geneva, Switzerland (SEROCoV-POP): a population-based study. Lancet. 2020;396(10247):313-319. doi:10.1016/S0140-6736(20)31304-0

4. Meyer B, Reimerink J, Torriani G, et al. Validation and clinical evaluation of a SARS-CoV-2 surrogate virus neutralisation test (sVNT). Emerging Microbes \& Infections. 2020;9(1):2394-2403. doi:10.1080/22221751.2020.1835448

5. R Core Team (2020). R: A Language and Environment for Statistical Computing. R Foundation for Satistical Computing https://www.R-project.org/

6. Stan Development Team (2020). RStan: The R Interface to Stan. http://mc-stan.org/

7. COVID-19 Situation Report. Accessed March 24, 2021. https://covidsitrep.moh.gov.sg/

8. Koo JR, Cook AR, Park M, et al. Interventions to mitigate early spread of SARS-CoV-2 in Singapore: a modelling study. The Lancet Infectious Diseases. Published online 2020.

9. Pollán M, Pérez-Gómez B, Pastor-Barriuso R, et al. Prevalence of SARS-CoV-2 in Spain (ENE-COVID): a nationwide, population-based seroepidemiological study. Lancet. 2020;396(10250):535-544. doi:10.1016/S0140-6736(20)31483-5

10. Steinberg J, Kennedy ED, Basler C, et al. COVID-19 Outbreak Among Employees at a Meat Processing Facility - South Dakota, March-April 2020. MMWR Morb Mortal Wkly Rep. 2020;69(31):1015-1019. 
doi:10.15585/mmwr.mm6931a2

11. Günther T, Czech-Sioli M, Indenbirken D, et al. SARS-CoV-2 outbreak investigation in a German meat processing plant. EMBO Mol Med. 2020;12(12):e13296. doi:10.15252/emmm.202013296

12. Middleton J, Reintjes R, Lopes $\mathrm{H}$. Meat plants-a new front line in the covid-19 pandemic. $B M J$. 2020;370:m2716. doi:10.1136/bmj.m2716

13. Spinelli MA, Glidden DV, Gennatas ED, et al. Importance of non-pharmaceutical interventions in lowering the viral inoculum to reduce susceptibility to infection by SARS-CoV-2 and potentially disease severity. The Lancet Infectious Diseases. Published online February 22, 2021. doi:10.1016/S1473-3099(20)30982-8

14. Emery JC, Russell TW, Liu Y, et al. The contribution of asymptomatic SARS-CoV-2 infections to transmission on the Diamond Princess cruise ship. Lipsitch M, Franco E, Lipsitch M, eds. eL ife. 2020;9:e58699. doi:10.7554/eLife.58699

\section{Tables}

Table 1: Distribution of age and underlying medical conditions in community cohort and migrant worker cohort participants, Singapore 2020 


\begin{tabular}{|c|c|c|}
\hline Characteristic & Community cohort $(\mathrm{N}=937)$ & Migrant worker cohort $(\mathrm{N}=478)$ \\
\hline \multicolumn{3}{|l|}{ Age (years) } \\
\hline - Mean (SD) & $52.3(13.0)$ & $35.2(7.9)$ \\
\hline - Range & $23.0-83.0$ & $19.0-59.0$ \\
\hline \multicolumn{3}{|l|}{ Age category } \\
\hline$-<30$ & $45(4.8 \%)$ & $134(28.0 \%)$ \\
\hline$-30-39$ & $114(12.2 \%)$ & $203(42.5 \%)$ \\
\hline$-40-49$ & $232(24.8 \%)$ & $124(25.9 \%)$ \\
\hline$-50-59$ & $270(28.8 \%)$ & $17(3.6 \%)$ \\
\hline$-60-69$ & $169(18.0 \%)$ & $0(0.0 \%)$ \\
\hline$-70+$ & $107(11.4 \%)$ & $0(0.0 \%)$ \\
\hline \multicolumn{3}{|c|}{ High blood pressure } \\
\hline - No & $718(76.7 \%)$ & $457(95.6 \%)$ \\
\hline - Yes & $217(23.2 \%)$ & $21(4.4 \%)$ \\
\hline - Don't know & $1(0.1 \%)$ & $0(0.0 \%)$ \\
\hline \multicolumn{3}{|l|}{ Heart disease } \\
\hline - No & $886(94.6 \%)$ & $478(100.0 \%)$ \\
\hline - Yes & $48(5.1 \%)$ & $0(0.0 \%)$ \\
\hline - Don't know & $3(0.3 \%)$ & $0(0.0 \%)$ \\
\hline \multicolumn{3}{|c|}{ Diabetes mellitus } \\
\hline - No & $794(84.8 \%)$ & $469(98.1 \%)$ \\
\hline - Yes & $140(15.0 \%)$ & $9(1.9 \%)$ \\
\hline - Don't know & $2(0.2 \%)$ & $0(0.0 \%)$ \\
\hline \multicolumn{3}{|c|}{ Chronic lung condition } \\
\hline - No & $873(93.2 \%)$ & $475(99.4 \%)$ \\
\hline - Yes & $61(6.5 \%)$ & $3(0.6 \%)$ \\
\hline - Don't know & $3(0.3 \%)$ & $0(0.0 \%)$ \\
\hline \multicolumn{3}{|l|}{ Smoking status } \\
\hline - Non-smoker & 792 (84.5\%) & 282 (59.0\%) \\
\hline
\end{tabular}




\begin{tabular}{|l|c|c|}
$-\quad$ Ex-smoker & $54(5.8 \%)$ & $46(9.6 \%)$ \\
\hline$-\quad$ Current smoker & $91(9.7 \%)$ & $150(31.4 \%)$ \\
\hline
\end{tabular}

\section{Figures}

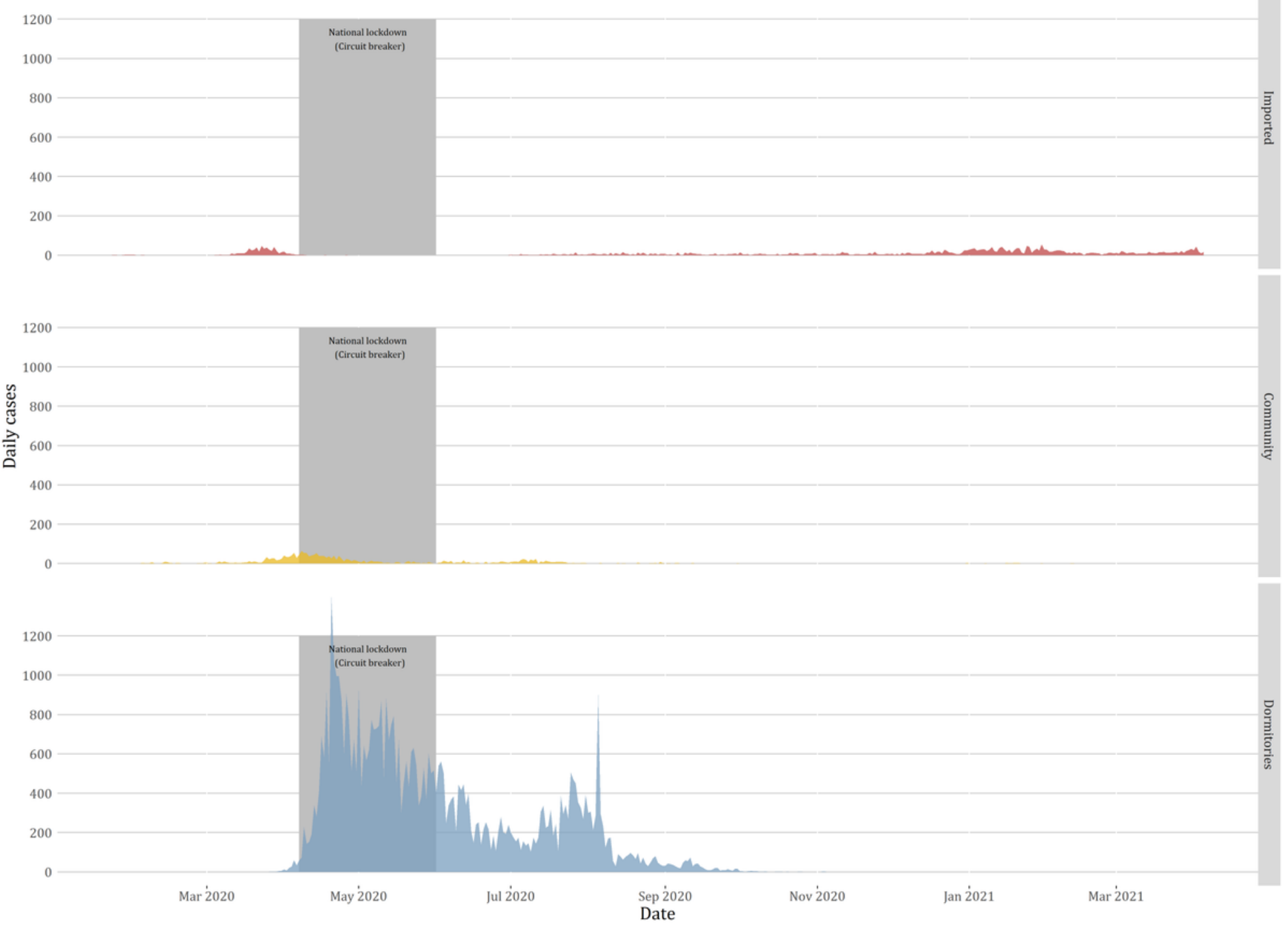

Figure 1

Epidemic patterns of imported (top panel), community (middle panel), and migrant worker dormitory (bottom panel) COVID-19 cases in Singapore, January 2020-March 2021. The shaded area represents the period of national lockdown (circuit breaker) between April 7 and June 1, 2020 

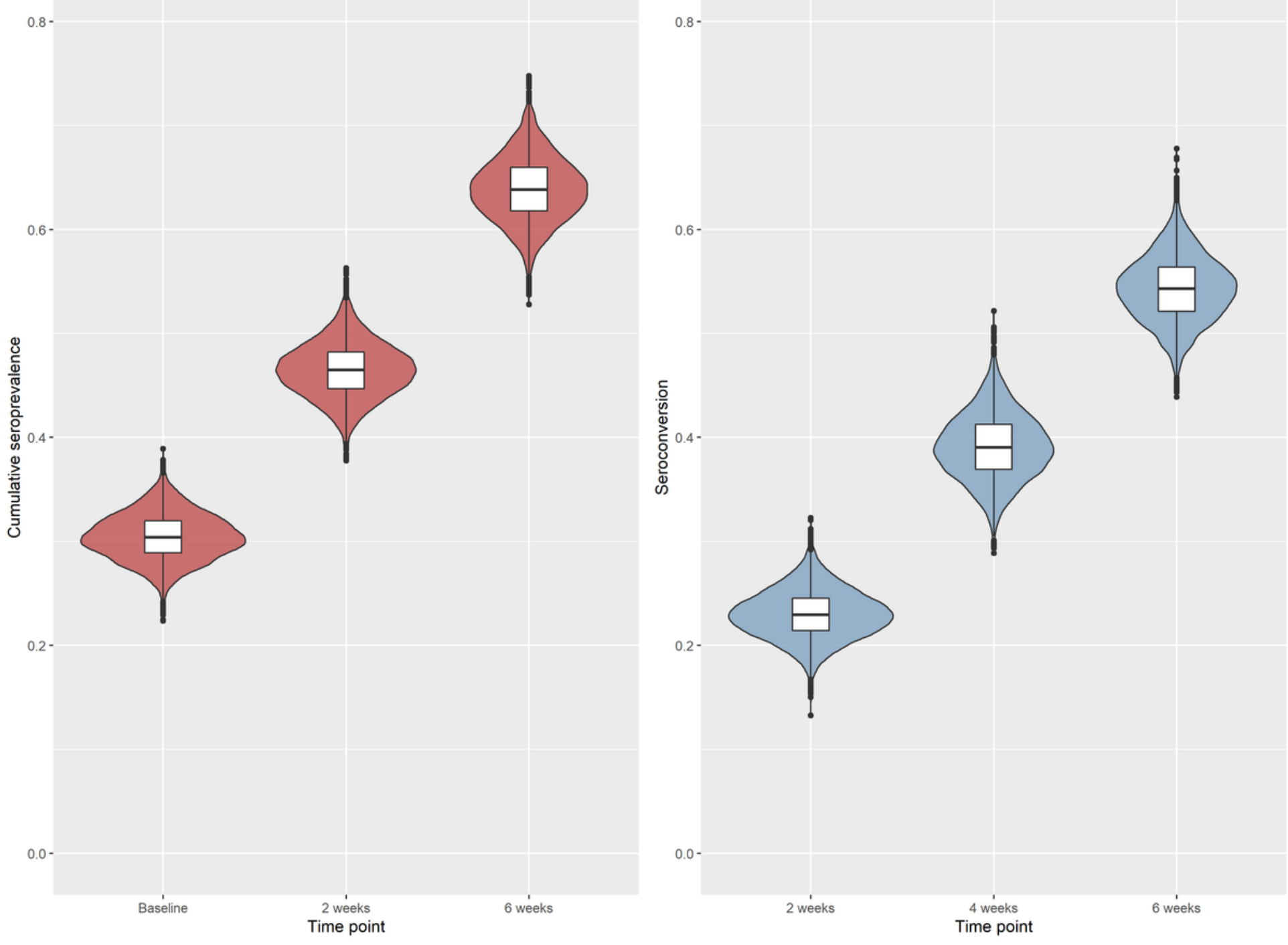

\section{Figure 2}

Cumulative seroprevalence (left panel) and seroconversion risk (right panel) over a six-week period among migrant workers residing in a dormitory, Singapore 2020. Two-week and six-week seroconversion estimates are based on antibody test results at two and six weeks among individuals initially seronegative at baseline. Four-week seroconversion estimates are based on antibody test results at the six-week follow-up among individuals who were seronegative at the two-week follow-up.

\section{Supplementary Files}

This is a list of supplementary files associated with this preprint. Click to download.

- wtseroprevalenceSupplement.docx

- KTDmapanimationcpass.gif 